CARNETS OE Carnets de géographes

GÉOGRAPHES.

$8 \mid 2015$

Géographie(s) de la lenteur

\title{
Tensions et compromis dans les valeurs spatiales du slow
}

\section{Mireille Diestchy}

\section{(2) OpenEdition}

\section{Journals}

Édition électronique

URL : http://journals.openedition.org/cdg/291

DOI : $10.4000 /$ cdg. 291

ISSN : 2107-7266

Éditeur

UMR 245 - CESSMA

Référence électronique

Mireille Diestchy, «Tensions et compromis dans les valeurs spatiales du slow », Carnets de géographes [En ligne], 8 | 2015, mis en ligne le 01 septembre 2015, consulté le 30 avril 2019. URL : http:// journals.openedition.org/cdg/291; DOl : 10.4000/cdg.291

Ce document a été généré automatiquement le 30 avril 2019

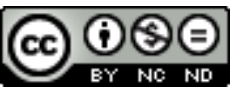

La revue Carnets de géographes est mise à disposition selon les termes de la Licence Creative Commons Attribution - Pas d'Utilisation Commerciale - Pas de Modification 4.0 International. 


\title{
Tensions et compromis dans les valeurs spatiales du slow
}

\author{
Mireille Diestchy
}

1 L'association internationale slow food naît en Italie en 1989. Elle est présente aujourd'hui dans plus de 150 pays et rassemble 80846 membres $^{1}$. Depuis la fin des années 2000, la dénomination slow a pris son autonomie: de nombreux collectifs européens et nordaméricains se sont emparés du terme pour marquer leur opposition à la fast life devenue, selon eux, la devise des modes de vie contemporains. Le terme se diffuse avec succès dans les domaines les plus divers du quotidien - de la recherche scientifique à l'éducation en passant par le tourisme et les médias - et tend même parfois à devenir un argument marketing ${ }^{2}$.

2 La diffusion de l'appellation slow recouvre, au-delà d'un simple effet de mode ou d'une aspiration à la seule lenteur, la revendication d'un ensemble de valeurs : des valeurs au caractère évident, telles que la « convivialité », la « solidarité » ou le " plaisir », et parfois contradictoires, lorsque la protection environnementale est articulée au "plaisir " personnel, par exemple. La difficulté d'analyse d'un tel objet est due à l'ambiguïté et à la polysémie de la terminologie «slow». Le terme est abondamment utilisé par les acteurs sans être aucunement défini et renvoie tout aussi bien à l'écologie, à la qualité, au calme ou à l'équitable, ce qui participe sans nul doute de son succès, car il peut faire écho à ces aspirations diverses. Il cristallise un système de valeurs ambivalentes, qu'il noue autour de la question temporelle, et dont l'élucidation permet de comprendre une aspiration contemporaine encore fort peu étudiée. Nous avons réalisé une enquête qualitative auprès des acteurs du slow dans le but d'analyser, de manière inductive, les registres de «valuations », au sens de John Dewey (Dewey, 2011), qui sous-tendent leur engagement. La théorie pragmatiste sur laquelle nous avons choisi de nous appuyer ${ }^{3}$, attentive à l'élaboration des jugements de valeur en situation, aux conflits et aux compromis esquissés par les acteurs, a mis en lumière de fortes tensions entre la valorisation de l'échelle locale et celle de l'échelle globale.

3 Une première partie présentera le terrain d'enquête et l'approche théorique adoptée. La seconde traitera des implications spatiales des «valuations » du slow. La tension entre 
local et global se décline sous deux formes: premièrement, si le slow rejoint en de nombreux points les processus contemporains de relocalisation des systèmes agroalimentaires, c'est moins la proximité géographique que la proximité relationnelle qui est valorisée; deuxièmement, une tension existe entre les diverses échelles de la portée de l'engagement, entre la promotion d'un plaisir personnel et l'engagement pour la protection de l'environnement. Les acteurs rencontrés tentent d'articuler ces aspirations ambivalentes et construisent dans ce but des compromis dont nous rendrons compte. Cette contribution propose ainsi une approche originale de la question spatiale : non pas une spatialisation des collectifs slow, mais une étude de l'espace en tant que représentation et valeur dans la promotion, aujourd'hui croissante, des modes de vie slow. L'analyse des tensions de valeurs permet, d'une part, de dépasser le consensus et l'apparente cohérence du phénomène ; d'autre part, d'observer comment les personnes articulent au quotidien des valeurs centrales de notre époque, mais qui peuvent entrer en contradiction : la valorisation de l'échelle locale pourvoyeuse de repères avec la richesse économique, culturelle et sociale de la mondialisation des échanges, et la quête personnelle du bonheur avec la prise de conscience environnementale.

\section{Enquêter sur les « valuations » du slow}

\section{Présentation des terrains d'enquête}

Notre enquête qualitative auprès des acteurs du slow entend contribuer à la compréhension d'un phénomène souvent mentionné, mais fort peu théorisé : beaucoup d'encarts dans la presse grand public, quelques essais anglophones et francophones qui proposent une analyse engagée, non scientifique de ce phénomène (Honoré, 2005; Andrews, 2008; Menétrey et Szerman, 2013). Des recherches en sciences sociales s'appuyant sur des enquêtes de terrain rigoureuses ont été menées (Leitch, 2003 ; Parkins et Craig, 2006; Siniscalchi, 2013; Deléage, 2014), mais elles portent sur slow food uniquement, pour en étudier l'histoire, le développement et les dynamiques organisationnelles. Dans le but de comprendre la diffusion du slow au-delà l'alimentation et de construire une définition transversale de ce terme, nous avons mené une enquête ethnographique, débutée en 2009 dans le cadre d'un master, au sein du Schnaeckele (petit escargot en alsacien), la structure bas-rhinoise, créée en 2006, de l'association internationale slow food. Nous avons complété cette observation par trente-cinq entretiens semi-directifs auprès de représentants de sept déclinaisons du slow. Ces dernières ont été choisies de manière à étudier les différentes formes prises par cette diffusion : des associations (slow food et slow cosmétique), des collectifs constitués autour de la publication d'un manifeste ou d'un ouvrage (slow management, slow design et slow science ), des références institutionnelles (slow art) et individuelles (slow cinéma).

5 L'histoire de slow food débute dans les années 1970 en Italie. De jeunes activistes de gauche, réunis autour de Carlo Petrini, le futur président de slow food, mènent alors des actions de valorisation du patrimoine alimentaire italien, du vin notamment. Ils sont affiliés à Arcigola (gola signifiant gourmandise), pendant alimentaire des associations populaires italiennes nommées ARCI (associations indépendantes de promotion culturelle). En 1986, Arcigola mène une campagne de protestation contre l'ouverture d'un McDonald's sur la piazza di Spagna, au centre de Rome. Le nom slow food émerge peu après et donne lieu à la rédaction d'un manifeste dont la signature, à Paris en 1989, 
marque la naissance de l'association internationale. slow food a pour slogan la promotion d'un aliment «bon, propre et juste ». Ces adjectifs rendent compte de la triangulation sur laquelle s'est construit le discours de l'association. L'anthropologue Valeria Siniscalchi souligne ainsi que «l'enchainement des trois termes correspond à l'évolution du mouvement et de sa philosophie au cours des quinze dernières années » : d'une attention à la qualité et au plaisir dans les années 1980 ; slow food a ensuite intégré la dimension écologique à la fin des années 1990, se renommant alors association "écogastronomique »; et enfin, la dimension équitable en mettant l'accent sur la juste rétribution des producteurs (Siniscalchi, $2013: 142$ ).

6 Émergent dès le milieu des années 2000, diverses déclinaisons du terme slow. En 2004, le designer britannique Alastair Fuad-Luke fait référence au slow design dans un texte intitulé slow theory et rédige quatre ans plus tard avec la chercheuse nord-américaine, Carolyn F. Strauss un manifeste posant les principes du slow design (Fuad-Luke et Strauss, 2008). Paraît, en 2010, un ouvrage intitulé Le slow management : éloge du bien-être au travail (Steiler, Sadowsky et Roche, 2010) écrit par trois universitaires. Prenant acte de l'augmentation des suicides dans le monde professionnel, un nouveau type de management est présenté qui repose sur une autre temporalité et un nouveau rapport entre manageurs et salariés. Dans le domaine de la recherche scientifique également, divers manifestes se réclamant de la slow science émergent entre les années 2010 et 2013. Les critiques portent sur les procédés d'évaluation et de mise en concurrence qui se diffusent dans le monde de la recherche scientifique et de l'enseignement supérieur. Ces derniers imposent une efficacité et une rentabilité qui sont autant de freins à la créativité et à la découverte, tributaires du temps long, des détours et des erreurs. Sur son site internet consacré à la cosmétique, le Belge Julien Kaibeck propose en 2011 la dénomination de slow cosmétique pour désigner une cosmétique « intelligente, raisonnée, écologique et humaine $»^{4}$. Structurée en association depuis 2012, la slow cosmétique réunit aujourd'hui des coordinateurs français, espagnols, italiens et belges. Slow art est le titre d'une exposition organisée par le musée national de Stockholm (Suède) en 2013 et présentée à Paris à l'institut suédois en mai 2014 (Robach, 2012). Elle réunit des œuvres ayant nécessité de longues heures de travail, réalisées hors commande et dans lesquelles sont intégrés et valorisés les aléas de la création. Enfin, le terme slow cinéma a été utilisé en 2014 par la cinéaste belge Martine Doyen sur son blog. Ayant découvert l'association slow food, elle explique que sa conception du cinéma reprend les mêmes exigences de qualité, de diversité, d'éducation et pourrait dès lors se nommer slow cinéma.

7 Il s'agissait pour nous de comprendre ce que peuvent avoir de commun ces formes très diverses de mobilisation du terme slow, attachées de plus à des domaines éloignés (la recherche scientifique et la cosmétique, par exemple). Les personnes partagent des caractéristiques socio-démographiques similaires: un haut niveau de diplôme (égal ou supérieur à $\mathrm{Bac}+5$ ) et catégories socioprofessionnelles «cadres et professions intellectuelles supérieures ", résidence en zone urbaine, en couple avec un ou plusieurs enfants et surreprésentation des 40-50 ans. Mais elles se rejoignent surtout sur un ensemble de revendications hétéroclites qui font système.

\section{Une approche pragmatiste des valeurs}

Dans l'optique d'étudier la manière dont les acteurs organisent et parviennent à concilier au quotidien des valeurs multiples et divergentes, nous nous appuyons sur la théorie 
pragmatiste de la "valuation» du philosophe américain John Dewey (1859-1952). Ce dernier propose une définition originale qui dépasse tant les théories subjectivistes (selon lesquelles la valeur est un "sentiment » éminemment individuel et n'est pas, de ce fait, un objet de science), que les théories rationalistes (attachées à une objectivité et à une rationalité des valeurs qui en font des principes abstraits, des a priori de l'action) : une conception de la valeur comme action processuelle, contextuelle et empiriquement observable. Le philosophe introduit la notion de "valuation " définie comme «ce à quoi nous tenons manifestement» (Dewey, $2011: 11$ ). Elle renvoie à l'action par laquelle nous attribuons de la valeur à quelque chose ou quelqu'un, en d'autres termes ce à quoi nous sommes attachés, ce que nous désirons obtenir et ce à quoi nous tentons de parvenir. « La valuation n'est pas l'enregistrement de valeurs existantes» (Bidet, 2008: 212), c'est un processus qui se construit dans l'expérience, émerge dans l'action et est donc fortement lié à la situation. Selon les contextes, un même individu peut être amené à valuer différents objets. Est ici remis en question l'a priori d'une cohérence du sujet en matière de valeurs, ce sont au contraire les acteurs eux-mêmes qui recherchent cette cohérence (Barthe et al., $2013: 191$ ).

9 Une telle théorie nous permet d'analyser de manière riche et fine les jugements de valeur, à la différence des enquêtes quantitatives qui sont pourtant l'outil principal, aujourd'hui, de la sociologie des valeurs. Les enquêtes statistiques sur les valeurs existent depuis les années 1970 aux États-Unis et en France depuis une vingtaine d'années. Ces méthodes procèdent par questionnaires; elles ont l'avantage d'être empiriques, non normatives et de prendre en compte la stratification sociale. Pour autant, elles présentent de nombreuses limites: le questionnaire ne porte pas sur des conduites effectives ou intentionnelles, mais sur des énoncés généraux hors contexte; il ne permet pas au chercheur d'observer l'activation des valeurs selon la situation (Heinich, 2006, 2010). L'observation directe et les entretiens semi-directifs, parce qu'ils sont le lieu d'un retour réflexif des acteurs sur leurs pratiques, permettent à l'inverse d'étudier les tensions et contradictions inhérentes aux opérations de " valuation ».

La notion pragmatiste de «valuation» fournit donc un cadre théorique pertinent pour analyser la dimension axiologique de la promotion du slow. Elle est articulée ici à la sociologie pragmatique française qui offre des outils précieux pour penser et conceptualiser la diversité des cadres normatifs. Cette approche postule une diversité des logiques d'action. Luc Boltanski et Laurent Thévenot ont construit le modèle des « cités ", six «mondes » auxquels sont attachées des grandeurs (ce qui est grand dans le monde) : le monde de l'inspiration (avec pour grandeurs, l'authenticité, la grâce), le monde domestique (la position dans un système de relations, la tradition, la confiance), le monde de l'opinion (le renom, la visibilité), le monde civique (l'intérêt général, le collectif), le monde marchand (le désirable, la concurrence) et le monde industriel (l'utilité, l'efficacité). À chacun de ces « mondes » correspond:

« un principe d'ordre différent permettant de spécifier de quoi est faite la grandeur des grands et, par là, de fonder un ordre justifiable entre les personnes. C'est sur de tels ordres que les personnes prennent appui lorsqu'elles doivent justifier leurs actions ou soutenir leurs critiques » (Boltanski et Thévenot, 1991 : 27-28).

Ce cadre d'analyse permet de catégoriser les multiples valeurs que recouvre le slow et d'étudier ce que revendiquent les acteurs. 


\section{Tensions et compromis dans les « valuations » du slow}

\section{De la valorisation du local à l'inscription dans une chaîne de dépendances personnelles}

11 Le slow, slow food principalement, entretient de fortes similitudes avec les stratégies contemporaines de relocalisation des processus de production, de distribution et de consommation. L'échelle locale est, en effet, perçue comme une garantie de protection environnementale, de justice sociale et de préservation de la diversité face au phénomène de mondialisation envisagé principalement comme vecteur d'uniformisation. Elle paraît être le lieu de l'embeddedness ${ }^{5}$ et de la liberté :

« Le localisme devient une contre-hégémonie à la thèse de la globalisation, un appel

à l'action au nom d'une lutte du pouvoir local contre le pouvoir global. En d'autres

termes, si le global est le lieu de la domination, le local, lui, permettrait de se libérer

${ }^{6} »$ (DuPuis et Goodman, $2005: 361$ ).

Pour Gianluca Brunori, le discours de l'association slow food associe la qualité à la diversité pour contrer les effets négatifs de l'industrialisation agroalimentaire (Brunori, 2007 : \$13). Le mouvement slow food, selon Sophie Dubuisson-Quellier, « vise à alerter le public contre l'homogénéisation des goûts et la disparition des cultures gastronomiques locales » (Dubuisson-Quellier, 2009 : 89). Il ferait ainsi la promotion d'une globalisation alternative, vertueuse et positive selon Geoff Andrews, journaliste ayant consacré un essai à l'association (Andrews, 2008: 152). Pour les membres de slow food rencontrés, consommer des produits locaux, mettre en avant « les valeurs du terroir» est un moyen de conserver la diversité et la spécificité du proche face à l'«homogénéisation » des produits de consommation. Il s'agit, dans un double mouvement, de valoriser le singulier face à la multiplication des échanges d'un marché devenu mondial ; tout en mettant en avant la variété, «la richesse» des identités locales face à des flux de biens et de personnes globalisés qui entrainent une standardisation des modes de vie. La condamnation de l'homogénéisation est présente dans les autres domaines du slow également: la cinéaste rencontrée explique que «tout devient un peu lisse ", tandis que les chercheurs de slow science condamnent le «formatage » des travaux scientifiques qui deviennent « des recettes de cuisine », «l'appauvrissement » et la «standardisation de la pensée ».

13 L'analyse des discours montre, cependant, que la valorisation du local est loin d'être constante. Le local peut aussi être synonyme d'isolement et de conservatisme, il est alors dévalué face à une échelle mondiale gage d'ouverture et de découverte :

«Ca dépend de jusqu'où on amène le local, il s'agit pas de se fermer... » [homme,

40-50 ans, cadre en entreprise puis restaurateur, Alsace, slow food].

De même, lors de la conférence slow art à l'institut culturel suédois de Paris, le fondateur du slow made évoque la centralité du local tout en soulignant que ça n'est pas un « repli sur soi identitaire », mais une "ouverture » autrement dit, explique-t-il, « une identité pas au sens négatif, mais au sens positif ». Cette expression imprécise montre bien l'effort déployé pour gérer l'ambivalence qui habite la valorisation du local, tentative vaine, car l'auditeur n'en sait guère plus sur cette identité. Mélanie E. Dupuis et David Goodman (2005) remettent en question les nombreux travaux scientifiques et discours militants qui proposent une conception positive des stratégies de relocalisation. La notion de «local» 
reste, selon eux, extrêmement floue et présente de nombreux risques : la formation d'une élite autoritaire et d'injustices sociales, la mise en place d'une politique «non-réflexive ", idéologique et réactionnaire.

La notion de local, au sens de proximité spatiale, ne nous permet donc pas de saisir de manière fine les "valuations" en jeu dans le slow et, plus largement, dans les phénomènes de relocalisation contemporains. Roos Gun, Terragni Laura et Torjusen Hanne soulignent la nécessité de redéfinir la notion de local dans le cadre actuel de la globalisation :

«Le local au sens d'une opposition au global, renvoie tant à la proximité qu'à des initiatives éloignées dans l'espace, qui deviennent "proches" en vertu de nouvelles relations d'interaction entre producteurs et consommateurs ${ }^{7} »$ (Roos, Terragni et Torjusen, 2007 : §3).

En ce sens, il qualifie moins la distance physique que la dimension relationnelle. Considérant que le terme de « local» entretient inéluctablement la confusion « entre les référents sociaux et spatiaux du local - le socialement proche et le géographiquement proche » (Deverre et Lamine, 2010:62) -, il convient de chercher plus avant comment caractériser cette valorisation de la proximité relationnelle qui est au cœur des stratégies de relocalisation contemporaines.

17 L'inscription dans des chaînes d'interdépendances, au-delà la proximité spatiale, permet de penser ensemble les contradictions liées à l'espace. La valorisation de l'inscription au sein d'une " chaîne de dépendances personnelles » est au cœur du « monde domestique » tel que le conceptualisent les chercheurs Luc Boltanski et Laurent Thévenot (1991). Dans le «monde domestique», est "grand» ce qui peut être situé dans un réseau d'interconnaissances. Le fait de pouvoir «mettre un visage derrière un produit », pour reprendre la formule des personnes rencontrées, offre une sensation de maîtrise et restaure la confiance. Une interviewée raconte sa relation avec un caviste :

«Il commençait ces derniers temps à nous recommander de plus en plus de vins naturels. Donc ça aussi c'était un peu nouveau pour nous, parce qu'on avait quelqu'un à qui on pouvait faire confiance » [femme, 50-60 ans, infirmière, Alsace, slow food].

18 Cette confiance est aujourd'hui mise à mal par des processus de dé-localisation. Anthony Giddens désigne ainsi le phénomène d'« extraction des relations sociales des contextes locaux d'interaction puis leur restructuration dans des champs spatio-temporels indéfinis » (Giddens, 1994 : 30). La dé-localisation favorise « les relations avec un autrui absent » (ibid : 27) et l'anonymat des objets, ce que dénonce le slow designer :

"On retrouve souvent dans nos maisons des objets anonymes, on n'a aucune idée de comment est-ce qu'ils ont été fabriqués " [homme, 30-40, designer, USA/ Vietnam, slow design].

19 Le direct dans ce cadre, plus que le local, rend compte des «valuations » du slow. Il fait référence à la réduction du nombre d'intermédiaires lors de la production et de l'acheminement. Et si le local est le plus souvent direct, le direct, quant à lui, n'est pas forcément local :

«On recrée un réseau de connaissances, on recrée des réseaux de distribution les plus propres possible, mais plus au niveau de son hameau, mais au niveau au moins de l'Europe, voire du monde" [homme, 40-50 ans, cadre en entreprise puis restaurateur, Alsace, slow food].

20 Lorsqu'un interviewé relate sa visite au "salon du goût ", une manifestation organisée par slow food tous les deux ans à Turin (Italie), il utilise une expression intéressante : « le 
monde est un village ». Le « monde » endosse ici les caractéristiques du " village » et de la familiarité rattachée au local parce que des gens se rencontrent et échangent en face à face. Des membres du convivium alsacien de slow food font venir des oranges de Sicile, cet échange, bien que s'effectuant sur une grande distance, a les caractéristiques du "direct », car l'intermédiaire est une personne connue. Au-delà l'hétérogénéité des discours slow, il est ainsi possible de rendre compte d'une critique formulée depuis le "monde domestique " - dans lequel sont valorisés les relations affectives et directes, l'entraide, le respect et la confiance - vers le "monde marchand» dans lequel les échanges sont régis par la recherche de profit et l'anonymat, et les personnes et les objets réduits à des « marchandises ».

\section{Entre l'intérêt de tous et le plaisir personnel}

La tension entre la valorisation du local et du global est nette également dans la perception que les promoteurs du slow ont de leur engagement, oscillant entre une recherche personnelle du plaisir et une lutte pour l'intérêt général. Comme l'ont montré Luc Boltanski et Laurent Thévenot, les acteurs formulant une critique ont l'impératif de "monter en généralité »: il s'agit de s'extraire des contingences et des parcours individuels pour montrer que la lutte relève de l'intérêt général et non de l'intérêt personnel. La légitimité du collectif et l'honnêteté de son combat dépendent ainsi d'une inscription dans le "monde civique", dans lequel "les citoyens renoncent à leurs singularités et se détachent de leurs intérêts particuliers pour ne regarder que le bien commun» (Boltanski et Thévenot, 1991: 138). C'est principalement sur le souci environnemental que les acteurs du slow construisent leur montée en généralité. Ils accusent les systèmes contemporains de production et de distribution de ne se soucier que de la productivité («monde industriel») et de l'intérêt financier d'une minorité ("monde marchand») au détriment de l'intérêt général. Le slow s'inscrit ainsi dans un processus plus large de construction de la question environnementale comme question sociale, qui émerge dans les années 1960 aux États-Unis et en Europe (Aspe et Jacqué, 2012). De manière surprenante, la notion d'« écologie » apparaît peu dans les discours. Les personnes rencontrées lui préfèrent le terme «slow» qui permet, selon elles, de souligner le caractère global de leur critique. L'écologie est en fait réduite par les enquêtés à la seule dimension de protection environnementale alors même que l'approche systémique est constitutive de l'idéologie écologiste (Dufoing, 2012 : 107).

Depuis le "monde civique ", les acteurs du slow dénoncent la poursuite d'un intérêt personnel pour délégitimer tant les autres déclinaisons du slow que des membres de leur propre collectif. Ainsi, le collectif d'universitaires belges qui mobilisait le terme de slow science à ses débuts l'a peu à peu abandonné au profit de la notion de "désexcellence ", car le terme slow est devenu, selon eux, une "mode » dont certains se saisissent pour générer du profit. Au sein de slow food également sont critiqués ceux qui ne chercheraient qu'un plaisir gustatif personnel, "les épicuriens", comparés aux «militants » qui s'engagent dans une lutte désintéressée.

Mais si les acteurs du slow affirment mener une lutte au nom de l'intérêt général, l'étude montre que leur engagement se limite principalement à la sphère privée. Les collectifs observés ne sollicitent que peu les institutions et les formes classiques du politique y sont dévalorisées (la manifestation ou l'engagement dans un parti, par exemple), les interviewés disent ne pas avoir un tempérament à «aller casser la vitrine comme José 
Bové » et «à faire des discours ou à se balader avec des pancartes ». Pour autant, leur action est tout de même présentée comme un combat " politique » : " manger est un acte politique » selon un slogan de slow food abondamment repris par les membres rencontrés. La notion de " politique » est alors utilisée au sens large et désigne « le travail déployé par les acteurs pour établir ou critiquer la légitimité des pouvoirs » (Dodier, 2003: 19). Elle rejoint la conception pragmatiste du travail "politique» au sens où les personnes contestent, au nom de certaines «valuations ", des relations de pouvoir existantes (Cefaï, 2009).

En tension avec cet engagement pour l'intérêt général, la notion de plaisir est au cœur des « valuations » du slow. Le plaisir gustatif est présent dès la création de slow food et la dimension sensorielle continue d'être centrale dans les déclinaisons du slow. La critique des modes de vie contemporains est alors justifiée depuis le "monde inspiré » dans lequel sont valorisées la subjectivité, l'émotion, la sensibilité. Dans le slow design et le slow management, la notion de bien-être vient s'ajouter à celle de plaisir. Cet accent mis sur le bien-être est à replacer dans le contexte plus large de valorisation du bonheur individuel, en France depuis les années 1960, décrit par l'historien Rémi Pawin (Pawin, 2013 : 55). Les personnes rencontrées dénoncent, au nom du plaisir, l'intransigeance de celui qui lutte pour la protection de l'environnement, désigné à de nombreuses reprises comme un « ayatollah » et la culpabilisation qui accompagnerait l'idéologie écologiste :

«C'est «bon, propre et juste » hein, propre oui, juste oui, mais il faut que ce soit bon aussi. Faut pas défendre corps et âme un truc... on n'est pas sado quoi [il rit] »

[homme, 40-50 ans, cadre dans une organisation européenne, Alsace, slow food].

Dans les travaux consacrés à slow food, l'articulation du plaisir et de l'écologie fait la spécificité de l'association: «Ce qui rend la philosophie de slow food unique est la fusion des concepts de gastronomie et d'écologie ${ }^{8} »$ (Andrews, $2008: 19$ ). Si l'association peut se prévaloir d'une approche "eco-gastronomique», comment les acteurs du slow parviennent-ils à articuler ces deux "valuations» au quotidien? Deux résolutions de cette tension entre le monde civique de l'engagement environnemental et le monde inspiré de la sensorialité ont pu être mises en lumière.

Premièrement, la modération leur permet de concilier le souci environnemental et le plaisir. Dans les entretiens apparaît la figure du «gourmet » en opposition à celle du "gourmand»: le plaisir valorisé dans le slow est mesuré; il peut être différé dans le temps, à la différence d'une jouissance immédiate qui caractériserait la société de consommation actuelle. Ce plaisir est lié à la satisfaction de «besoins » supposés " essentiels ", et à un détachement vis-à-vis de "désirs " "superficiels ", rejoignant la conception philosophique du bonheur chez Épicure. Le plaisir de chacun est ainsi intimement lié à la collectivité au sens où le plaisir individuel peut être reporté et limité par les besoins du collectif. Est promu par un interviewé « le plaisir qui dure » qui prend en compte la question de la durabilité des ressources. Ce plaisir borné par l'intérêt général procure de la satisfaction: «la contrainte est structurante », comme le souligne un membre de slow food. Dans un article consacré à la notion de plaisir dans la presse féminine, la sociologue Faustine Régnier observe que le plaisir est de plus en plus lié à la nécessité : « l'analyse des domaines d'application du plaisir montre le passage d'un plaisir opposé à la contrainte à un plaisir qui se situe désormais dans le respect de la contrainte » (Régnier, 2014 : 102). Dans le même temps, il s'agit bien de ne pas sacrifier le plaisir individuel à la collectivité, car du plaisir de chacun dépend le plaisir de tous. L'expression "droit au plaisir », présente chez slow food et reprise par les interviewés, rend bien 
compte de cette tentative de résolution entre la collectivité et le personnel, car le plaisir est élevé au rang de droit qui est une grandeur du « monde civique ».

Le compromis s'esquisse également entre l'attention au plaisir quotidien et l'engagement pour un changement de société. La vision du changement social permet aux acteurs d'articuler leurs pratiques quotidiennes aux enjeux économiques et environnementaux mondiaux. Cela, alors même qu'ils soulignent le peu d'impact de leur comportement individuel sur ces enjeux. Cette tension est résolue par l'idée abondamment citée que "chacun doit faire sa part " et que la société peut changer si chacun "agit à son échelle»:

«On peut agir par sa propre action, on peut contribuer même petitement à changer le cours des choses " [homme, 50-60 ans, enseignant-chercheur, Belgique, slow science /Désexcellence].

Ce principe de la portée globale des actions locales est présent plus largement dans les mouvements environnementaux :

«les formes de contestation contemporaine trouvent leur source dans cette approche interactive avec une amplification de l'acte individuel comme acte militant " qui se résume par la formule " penser globalement, agir localement " (Aspe et Jacqué, $2012: 34$ ).

Les personnes rencontrées mettent en avant l'importance de traduire leurs valeurs dans leurs actions quotidiennes, ce qu'ils appellent avancer par " petits pas ». C'est ainsi que le changement social commence, selon eux, par une transformation de soi-même :

«Et donc publier des livres, des articles, parler [à propos de la slow science, de la désexcellence], oui bien sûr il faut le faire. Mais ce qui est important aussi c'est de transformer nos vies ici et maintenant " [homme, 50-60 ans, enseignant-chercheur, Belgique, slow science /Désexcellence].

\section{Conclusion}

30 Le phénomène de diffusion du slow pourrait être envisagé quelque peu rapidement comme un appel au ralentissement associé à une critique de la mondialisation. Il se traduirait alors, dans les discours et pratiques de ceux qui s'en revendiquent, par une valorisation et un repli sur l'échelle locale. L'enquête auprès des acteurs du slow met en lumière une réalité plus complexe, marquée par des tensions de "valuations » et des compromis. Ainsi, le slow, bien que rejoignant les initiatives contemporaines de relocalisation des circuits agroalimentaires, ne peut être réduit à la promotion d'une échelle locale avec laquelle les personnes rencontrées entretiennent un rapport ambivalent. L'enquête montre que, plus que la proximité spatiale, c'est la proximité relationnelle, en d'autres termes, l'inscription dans un réseau d'interconnaissances, qui est au cœur des «valuations» du slow. De même, le slow entretient de fortes similitudes avec les mouvements environnementaux, mais il ne peut être envisagé que sous cette seule dimension: le plaisir et la subjectivité sont également centraux dans les « valuations » étudiées. Les acteurs articulent alors les échelles du local, de l'individuel et du quotidien avec celles du collectif, de l'intérêt général et de l'environnement naturel dans sa totalité, au travers la formation de compromis : le "plaisir qui dure », le « droit au plaisir » et un changement social résultant des « petits pas » de chacun.

Le slow est envisagé ici comme la revendication d'un ensemble de "valuations » qui font système et qui ont pour particularité de se nouer sur la question rythmique. C'est, en 
effet, par l'adoption de rythmes de vie spécifiques que les acteurs entendent pouvoir suivre ces valeurs. Nous ne pourrons développer, dans le volume qui nous est imparti ici, la traduction de ces "valuations» dans les temporalités quotidiennes des interviewés. Notons toutefois qu'analyser le slow comme une aspiration à la lenteur n'est pas satisfaisant: la notion reste largement dévalorisée sur le terrain et le slow renvoie davantage, pour les personnes rencontrées, à l'expression "prendre le temps». Le sociologue William Grossin souligne également la nécessité de dépasser l'opposition lenteur-vitesse pour analyser les évolutions des temporalités contemporaines (Grossin, 2000). Le recours à la dénomination slow traduit, plus qu'une aspiration au ralentissement, la volonté de prendre le pouvoir sur les rythmes contemporains, individuels et collectifs et de choisir ainsi les modalités de vécu du temps quotidien. La critique slow participe ainsi d'une problématisation des rythmes économiques et sociaux qui deviennent une question d'intérêt général, un « problème public » (Cefaï, 1996).

Cependant, à cette publicisation de la question temporelle, il semble que les personnes proposent une réponse centrée en grande partie sur l'individu. Le slow renvoie, en effet, à l'adoption d'une éthique personnelle, à un travail sur soi, proche du "souci de soi " étudié par Michel Foucault (Foucault, 1984). Cette éthique est source de subjectivation, au sens où elle participe d'un sentiment de maîtrise, mais elle est dans le même temps vecteur d'assujettissement. Les acteurs du slow modifient leurs modes de vie pour répondre aux défis globaux et notamment à la menace que constitue le changement climatique. Or, les modes de gouvernements tendent aujourd'hui à s'appuyer sur ce sentiment de responsabilité individuelle et, plus encore, à le promouvoir, dans le but de reporter sur l'individu des nécessités de changement qui incomberaient à la collectivité. Cette gouvernance centrée sur la responsabilisation, propre au néolibéralisme (Hache, 2007), s'appuie sur des « politiques environnementales [qui] en appellent de plus en plus directement à la responsabilité des individus dans leurs différentes figures (usagercitoyen-consommateur-électeur-contribuable...) pour les amener à contribuer personnellement à la régulation des problèmes collectifs» (Salles, 2009 : §17). Ce report est d'autant plus efficace que l'autonomie est aujourd'hui revendiquée et socialement valorisée (Ehrenberg, 2005).

\section{BIBLIOGRAPHIE}

ANDREWS G. (2008), The slow food story. Politics and pleasure, Montreal, McGill-Queen's University Press, 196p.

ASPE C., JACQUE M. (2012), Environnement et société : une analyse sociologique de la question environnementale, Paris, MSH, 279p.

BARTHE Y. et al. (2013) « Sociologie pragmatique : mode d'emploi », Politix, 2013/3, pp.175-204.

BIDET A. (2008) « La genèse des valeurs : une affaire d'enquête », Tracés, n 15, pp.217-28. http://traces.revues.org/813, consulté le 21 octobre 2014. 
BOLTANSKI L., THEVENOT L. (1991), De la justification : les économies de la grandeur, Paris, Gallimard, 483p.

BRUNORI G. (2007) « Local Food and Alternative Food Networks: A Communication Perspective », Anthropology of Food, $\mathrm{n}^{\circ} \mathrm{S} 2$.

http://aof.revues.org/430?lang=fr, consulté le 19 janvier 2015.

CEFAÏ D. (1996) « La construction des problèmes publics. Définitions de situations dans des arènes publiques », Réseaux, 14 (75), pp.43-66.

http://www.persee.fr/web/revues/home/prescript/article/ reso_0751-7971_1996_num_14_75_3684, consulté le 19 février 2015.

CEFAÏ D. (2009) « Comment se mobilise-t-on? L'apport d'une approche pragmatiste à la sociologie de l'action collective ", Sociologie et sociétés, 2009/2, pp.245-69.

http://www.erudit.org/revue/SOCSOC/2009/v41/n2/039267ar.html, consulté le 13 janvier 2015.

DELEAGE E. (2014) « Le mouvement Slow Food : contretemps de l'accélération temporelle?», Ecologie \& politique, 2014/1, pp.49-59.

DEVERRE C., LAMINE C. (2010) «Les systèmes agroalimentaires alternatifs. Une revue de travaux anglophones en sciences sociales », Economie rurale, 2010/3, pp.57-73.

DEWEY J. (2011), La formation des valeurs, Paris, La Découverte, 234p.

DODIER N. (2003), Leçons politiques de l'épidémie de sida, Paris, Éditions de l'EHESS, 359p.

DUBUISSON-QUELLIER S. (2009), La consommation engagée, Paris, Sciences Po, 143p.

DUFOING F. (2012), L'écologie radicale, Gollion, Infolio, 157p.

DUPUIS E. M., GOODMAN D. (2005) «Should We Go "home" to Eat?: Toward a Reflexive Politics of Localism », Journal of Rural Studies, 2005/3, pp.359-71.

EHRENBERG A. (2005) « Agir de soi-même », Esprit, juillet, pp.200-209.

FOUCAULT M. (1984), Histoire de la sexualité 2. L'usage des plaisirs, Paris, Gallimard, 339p.

FUAD-LUKE A., STRAUSS C. F. (2008) « The Slow Design Principles », Slow Lab, Amsterdam. http://www.slowlab.net/CtC_SlowDesignPrinciples.pdf, consulté le 21 octobre 2014.

GRANOVETTER M. (1985) «Economic Action and Social Structure: The Problem of embeddedness ", American Journal of Sociology, 1985/3, pp.481-510.

GIDDENS A. (1994), Les conséquences de la modernité, Paris, L’Harmattan, 192p.

GROSSIN W. (2000) «Les bienfaits de la lenteur », Social Science Information, 2000/2, pp.219-32.

HACHE E. (2007) « La responsabilité, une technique de gouvernementalité néolibérale ? », Raisons politiques, 2007/4, pp.49-65.

http://www.cairn.info/revue-raisons-politiques-2007-4-page-49.htm, consulté le 12 février 2015.

HEINICH N. (2006) « La sociologie à l'épreuve des valeurs », Cahiers internationaux de sociologie, 2006/2, pp.287-315.

http://www.cairn.info/revue-cahiers-internationaux-de-sociologie-2006-2-page-287.htm, consulté le 23 mai 2014.

HEINICH N. (2010) « Note sur les méthodes quantitatives en sociologie des valeurs », Bulletin de Méthodologie sociologique, $\mathrm{n}^{\circ}$ 108, pp.5-13.

HONORÉ C. (2005), In praise of slowness: challenging the cult of speed, San Francisco, Harper San Francisco, 321p. 
LEITCH A. (2003) «Slow food and the politics of pork fat: Italian food and European identity », Ethnos, 2003/4, pp.437-62.

LE VELLY R. (2002) « La notion d'encastrement : une sociologie des échanges marchands », Sociologie du travail, 2002/1, pp.37-53.

MENETREY S., SZERMAN S. (2013), Slow attitude ! : oser ralentir pour mieux vivre, Paris, Armand Colin, 255p.

MONDEME T., LAVERGNE C. (2008) « Pragmatismes : vers une politique de l'action située », Tracés. Revue de sciences humaines, $\mathrm{n}^{\circ} 15$.

http://traces.revues.org/583, consulté le 10 octobre 2012.

OGIEN A. (2014) « Pragmatismes et sociologies », Revue française de sociologie, 2014/3, pp.563-579.

PARKINS W., CRAIG G. (2006), Slow living, Oxford, Berg, 180p.

PAWIN R. (2013), Histoire du bonheur en France depuis 1945, Paris, R. Laffont, 379p.

POLANYI K. (2009), La grande transformation : aux origines politiques et économiques de notre temps, Paris, Gallimard, (1944 pour la première édition), 467p.

REGNIER F. (2014) «L'alimentation entre plaisir(s) et nécessité(s) en France et aux États-Unis : Quelques variations dans la presse féminine depuis les années 1930 », Sociologie et sociétés, 2014/2, pp.85-108.

ROBACH C. (2012), Catalogue de l'exposition Slow Art, Stockholm, Nationalmuseum, 170p. http://www.nationalmuseum.se/Global/Publikationer/NM_SlowArt_digital_fix_eng.pdf, consulté le 21 octobre 2014.

ROOS G., TERRAGNI L., TORJUSEN H. (2007) « The Local in the Global - Creating Ethical Relations between Producers and Consumers ", Anthropology of Food, $\mathrm{n}^{\circ}$ S2.

http://aof.revues.org/489?lang=fr, consulté le 19 janvier 2015

SALLES D. (2009) « Environnement : la gouvernance par la responsabilité ? ", VertigO - la revue électronique en sciences de l'environnement, Hors série 6.

http://vertigo.revues.org/9179, consulté le 21 juillet 2014.

SIMMEL G. (1987), Philosophie de l'argent, Paris, PUF, (1900 pour la première édition), 662p.

SINISCALCHI V. (2013) «Slow versus fast ». Terrain. Revue d'ethnologie de l'Europe, $\mathrm{n}^{\circ}$ 60, pp.132-47.

http://terrain.revues.org/15122, consulté le 14 mars 2013.

STAVO-DEBAUGE J. (2012) «La sociologie dite "pragmatique" et la philosophie pragmatiste, une rencontre tardive », Séminaire, Villa Vigoni, Italie.

STEILER D., SADOWSKY J., ROCHE L. (2010), Le slow management : éloge du bien-être au travail, Grenoble, Presses universitaires de Grenoble, 105p.

WEBER M. (1995), Économie et société, Vol. 1. Paris, Pocket, (1956 et 1967 pour les premières éditions en langue allemande), 410p.

\section{NOTES}

1. Chiffres au 31 décembre 2012, cf. Almanach Slow Food, 2013, p.130.

2. La marque Bacardi Martini France, par exemple, recourt à l'expression slow drinking. 
3. Le terme « pragmatiste » fait référence ici à la fois au courant de philosophie américaine de la fin du XIXe siècle (Ogien, 2014), notamment à la «théorie de la valuation » (Dewey, 2011) ; et à la sociologie pragmatique française de la fin du $\mathrm{XX}^{\mathrm{e}}$ siècle, voir les travaux de Luc Boltanski et Laurent Thévenot (Boltanski et Thévenot, 1991). Ces deux approches ne sont pas directement affiliées (Mondémé et Lavergne, 2008) mais partagent un certain nombre de "schèmes" communs (Stavo-Debauge, 2012), du fait principalement de l'héritage de l'interactionnisme symbolique et de l'ethnométhodologie.

4. Site « L'essentiel de Julien »

http://www.lessentieldejulien.com/2011/09/la-slow-cosmetique-revenir-a-lessentiel/, saisi le $21 / 10 / 2014$.

5. L'« embededdness » ou « encastrement » désigne l'inscription des échanges marchands dans un contexte social dans lequel interviennent des normes et des valeurs, des sentiments psychosociaux (confiance), une culture matérielle (monnaie), etc. En introduisant cette notion, Mark Granovetter (1985) et, plus récemment, Karl Polanyi (2009), soulignent que « l'économique est encastré dans le social» (Le Velly, 2002: 38). Des auteurs classiques de la sociologie, Max Weber (1995) ou Georg Simmel (1987) soulignaient déjà au début du XX $\mathrm{XX}^{\mathrm{e}}$ siècle les dimensions sociales et symboliques des échanges économiques.

6. « localism becomes a counter-hegemony to this globalization thesis, a call to action under the claim that the counter to global power is local power. In other words, if global is domination then in the local we must find freedom ".

7. «local, when seen as an opposition to global, may imply nearness to geographically distant initiatives, which become "close" by virtue of new kinds of relations interconnecting producers and consumers ».

8. "What makes Slow Food's philosophy unique is the amalgamation of the two concept of gastronomy and ecology ».

\section{RÉSUMÉS}

La dénomination slow, apparue à la fin des années 1980 avec la création de slow food, se diffuse aujourd'hui dans le monde occidental dans de multiples domaines de la vie quotidienne. L'appellation slow recouvre selon nous, au-delà d'un simple effet de mode ou d'une aspiration à la seule lenteur, la revendication d'un ensemble de valeurs symptomatiques d'une remise en question des rythmes contemporains. Au cœur de ces dernières, nous avons mis en lumière une tension entre local et global. Les acteurs du slow tentent d'articuler ces aspirations ambivalentes et construisent dans ce but des compromis. Cette contribution propose ainsi une approche originale de la question spatiale: non pas une spatialisation des collectifs slow, mais une étude de l'espace en tant que représentation et valeur dans la promotion, aujourd'hui croissante, des modes de vie slow.

The slow name, appeared in the late 1980s with the creation of slow food, spreads in the Western world today in many areas of everyday life. More than a promotion of slowness or a fad phenomenon, the slow reveals the demand of a set of values. We have highlighted a tension between local and global within these values. The slow members try to articulate these ambivalent aspirations and build compromises. In this paper, we propose a novel approach of the question of space: not a spatialization of slow groups, but a study of space as representation and value in the increasing promotion of slow lifestyles. 
INDEX

Thèmes: Carnets de recherches

\section{AUTEUR}

\section{MIREILLE DIESTCHY}

I3 UMR 9217 - CNRS - Télécom Paris-Tech - Université Paris-Saclay

Sociologie

mireille.diestchy[at]gmail.com 